\title{
On a multiple Hilbert-type integral inequality involving the upper limit functions
}

Jianhua Zhong ${ }^{1 *}$ and Bicheng Yang

"Correspondence: jajones@163.com 'Department of Mathematics, Guangdong University of Education, Guangzhou, Guangdong 51003, China

\section{Springer}

\begin{abstract}
By applying the weight functions, the idea of introducing parameters and the technique of real analysis, a new multiple Hilbert-type integral inequality involving the upper limit functions is given. The constant factor related to the gamma function is proved to be the best possible in a condition. A corollary about the case of the nonhomogeneous kernel and some particular inequalities are obtained.
\end{abstract}

MSC: $26 \mathrm{D} 15$

Keywords: Weight function; Hilbert-type integral inequality; Upper limit function; Parameter; Gamma function

\section{Introduction}

Assuming that $0<\sum_{m=1}^{\infty} a_{m}^{2}<\infty$ and $0<\sum_{n=1}^{\infty} b_{n}^{2}<\infty$, we have the following Hilbert's inequality with the best possible constant factor $\pi$ (cf. [1], Theorem 315):

$$
\sum_{m=1}^{\infty} \sum_{n=1}^{\infty} \frac{a_{m} b_{n}}{m+n}<\pi\left(\sum_{m=1}^{\infty} a_{m}^{2} \sum_{n=1}^{\infty} b_{n}^{2}\right)^{1 / 2} .
$$

If $0<\int_{0}^{\infty} f^{2}(x) d x<\infty$ and $0<\int_{0}^{\infty} g^{2}(y) d y<\infty$, then we still have the following integral analogue of (1), named Hilbert's integral inequality (cf. [1], Theorem 316):

$$
\int_{0}^{\infty} \int_{0}^{\infty} \frac{f(x) g(y)}{x+y} d x d y<\pi\left(\int_{0}^{\infty} f^{2}(x) d x \int_{0}^{\infty} g^{2}(y) d y\right)^{1 / 2}
$$

where the constant factor $\pi$ is the best possible. Inequalities (1) and (2) play an important role in analysis and its applications (cf. [2-13]).

The following half-discrete Hilbert-type inequality was provided: If $K(x)(x>0)$ is a decreasing function, $p>1, \frac{1}{p}+\frac{1}{q}=1,0<\phi(s)=\int_{0}^{\infty} K(x) x^{s-1} d x<\infty, f(x) \geq 0,0<$ $\int_{0}^{\infty} f^{p}(x) d x<\infty$, then (cf. [1], Theorem 351)

$$
\sum_{n=1}^{\infty} n^{p-2}\left(\int_{0}^{\infty} K(n x) f(x) d x\right)^{p}<\phi^{p}\left(\frac{1}{q}\right) \int_{0}^{\infty} f^{p}(x) d x
$$

(c) The Author(s) 2021. This article is licensed under a Creative Commons Attribution 4.0 International License, which permits use, sharing, adaptation, distribution and reproduction in any medium or format, as long as you give appropriate credit to the original author(s) and the source, provide a link to the Creative Commons licence, and indicate if changes were made. The images or other third party material in this article are included in the article's Creative Commons licence, unless indicated otherwise in a credit line to the material. If material is not included in the article's Creative Commons licence and your intended use is not permitted by statutory regulation or exceeds the permitted use, you will need to obtain permission directly from the copyright holder. To view a copy of this licence, visit http://creativecommons.org/licenses/by/4.0/. 
In recent years, some new extensions of (3) were provided by [14-19].

In 2006, by using Euler-Maclaurin summation formula, Krnic et al. [20] gave an extension of (1) with the kernel $\frac{1}{(m+n)^{\lambda}}(0<\lambda \leq 4)$. In 2019, following the result of [20], Adiyasuren et al. [21] considered an extension of (1) involving the partial sums. In 2016-2017, by applying the weight functions, Hong $[22,23]$ obtained some equivalent statements of the extensions of (1) and (2) with a few parameters. A few similar works were provided by [24-38].

In this paper, following the idea of [21], by using the weight functions, the way of introducing parameters and the technique of real analysis, a new multiple Hilbert-type integral inequality with the kernel $\frac{1}{\left(x_{1}+\cdots+x_{n}\right)^{\lambda}}(\lambda>0)$ involving the upper limit functions is given. The constant factor related to the gamma function is proved to be the best possible in a condition. A corollary about the case of the nonhomogeneous kernel and some particular inequalities are obtained.

\section{Some lemmas}

In what follows, we assume that $n \in \mathrm{N} \backslash\{1\}:=\{2,3, \ldots\}, p_{i}, r_{i}>1(i=1, \ldots, n), \sum_{i=1}^{n} \frac{1}{p_{i}}=$ $1, \lambda>0, c_{\lambda}:=\left(1-\sum_{j=1}^{n} \frac{1}{r_{j}}\right) \lambda, f_{i}(x)(i=1, \ldots, n)$ are nonnegative measurable functions in $R_{+}=(0, \infty)$ such that $f_{i}(x)=o\left(e^{t x}\right)(t>0 ; x \rightarrow \infty)$, and for any $A=(0, a)(a>0), f_{i} \in L^{1}(A)$, the upper limit functions are defined by $F_{i}(x):=\int_{0}^{x} f_{i}(t) d t(x \geq 0)$, satisfying

$$
0<\int_{0}^{\infty} x_{i}{ }^{-p_{i} \frac{\lambda}{r_{i}}-c_{\lambda}-1} F_{i}^{p_{i}}\left(x_{i}\right) d x_{i}<\infty \quad(i=1, \ldots, n) .
$$

By the definition of the gamma function, for $x_{i}>0(i=1, \ldots, n)$, the following expression holds:

$$
\frac{1}{\left(\sum_{i=1}^{n} x_{i}\right)^{\lambda}}=\frac{1}{\Gamma(\lambda)} \int_{0}^{\infty} t^{\lambda-1} e^{-t \sum_{i=1}^{n} x_{i}} d t
$$

Lemma 1 For $t>0$, we have the following expressions:

$$
\int_{0}^{\infty} e^{-t x} f_{i}(x) d x=t \int_{0}^{\infty} e^{-t x} F_{i}(x) d x \quad(i=, 1, \ldots, n)
$$

Proof In view of $F_{i}(0)=0$,we find

$$
\begin{aligned}
\int_{0}^{\infty} e^{-t x} f_{i}(x) d x & =\int_{0}^{\infty} e^{-t x} d F_{i}(x) \\
& =\left.e^{-t x} F_{i}(x)\right|_{0} ^{\infty}-\int_{0}^{\infty} F_{i}(x) d e^{-t x} \\
& =\lim _{x \rightarrow \infty} \frac{F_{i}(x)}{e^{t x}}+t \int_{0}^{\infty} e^{-t x} F_{i}(x) d x .
\end{aligned}
$$

If $F_{i}(\infty)=$ constant, then $\lim _{x \rightarrow \infty} \frac{F_{i}(x)}{e^{t x}}=0$ and (5) follows; if $F_{i}(\infty)=\infty$, since $f_{i}(x)=$ $o\left(e^{t x}\right)(x \rightarrow \infty)$, we find

$$
\int_{0}^{\infty} e^{-t x} f_{i}(x) d x=\lim _{x \rightarrow \infty} \frac{F_{i}^{\prime}(x)}{\left(e^{t x}\right)_{x}^{\prime}}+t \int_{0}^{\infty} e^{-t x} F_{i}(x) d x
$$




$$
=\lim _{x \rightarrow \infty} \frac{f_{i}(x)}{t e^{t x}}+t \int_{0}^{\infty} e^{-t x} F_{i}(x) d x=0+t \int_{0}^{\infty} e^{-t x} F_{i}(x) d x
$$

and then (5) follows, too.

The lemma is proved.

Lemma 2 For $x_{i}>0(i=1, \ldots, n)$, the following expression holds:

$$
A:=\prod_{i=1}^{n}\left[x_{i}^{\left(\frac{\lambda}{r_{i}}-1\right)\left(1-p_{i}\right)} \prod_{j=1(j \neq i)}^{n} x_{j}^{\frac{\lambda}{r_{j}}-1}\right]^{\frac{1}{p_{i}}}=1
$$

Proof We have

$$
\begin{aligned}
A & =\prod_{i=1}^{n}\left[x_{i}^{\left(\frac{\lambda}{r_{i}}-1\right)\left(1-p_{i}\right)+1-\frac{\lambda}{r_{i}}} \prod_{j=1}^{n} x_{j}^{\frac{\lambda}{r_{j}}-1}\right]^{\frac{1}{p_{i}}}=\prod_{i=1}^{n}\left[x_{i}^{\left(\frac{\lambda}{r_{i}}-1\right)\left(-p_{i}\right)}\right]^{\frac{1}{p_{i}}}\left(\prod_{j=1}^{n} x_{j}^{\frac{\lambda}{r_{j}}-1}\right)^{\frac{1}{p_{i}}} \\
& =\prod_{i=1}^{n} x_{i}^{1-\frac{\lambda}{r_{i}}}\left(\prod_{j=1}^{n} x_{j}^{\frac{\lambda}{r_{j}}-1}\right)^{\sum_{i=1}^{n} \frac{1}{p_{i}}}=\prod_{i=1}^{n} x_{i}^{1-\frac{\lambda}{r_{i}}}\left(\prod_{j=1}^{n} x_{j}^{\frac{\lambda}{r_{j}}-1}\right)=1
\end{aligned}
$$

and then (6) follows.

The lemma is proved.

Lemma 3 For $n \in \mathrm{N} \backslash\{1\}$, defining the following weight functions:

$$
\omega_{\lambda}^{(i)}\left(x_{i}\right):=x_{i}^{\frac{\lambda}{r_{i}}+c_{\lambda}} \int_{0}^{\infty} \cdots \int_{0}^{\infty} \frac{1}{\left(\sum_{i=1}^{n} x_{i}\right)^{\lambda}} \prod_{j=1(j \neq i)}^{n} x_{j}^{\frac{\lambda}{r_{j}}-1} d x_{1} \cdots d x_{i-1} d x_{i+1} \cdots d x_{n},
$$

we have

$$
\omega_{\lambda}^{(i)}\left(x_{i}\right)=k_{\lambda}^{(i)}:=\frac{\Gamma\left(\lambda\left(1-\frac{1}{r_{i}}\right)\right)}{\Gamma\left(\sum_{j=1(j \neq i)}^{n} \frac{\lambda}{r_{j}}\right)} \cdot \frac{\prod_{j=1}^{n} \Gamma\left(\frac{\lambda}{r_{j}}\right)}{\Gamma(\lambda)} \in \mathrm{R}_{+} \quad(i=1, \ldots, n) .
$$

In particular, for $\sum_{i=1}^{n} \frac{1}{r_{i}}=1$, we have

$$
k_{\lambda}^{(i)}=k_{\lambda}:=\frac{1}{\Gamma(\lambda)} \prod_{j=1}^{n} \Gamma\left(\frac{\lambda}{r_{j}}\right) \quad(i=1, \ldots, n) .
$$

Proof For $j \neq i$, setting $u_{j}=\frac{x_{j}}{x_{i}}$ in (7), we have

$$
\begin{aligned}
\omega_{\lambda}^{(i)}\left(x_{i}\right)= & \int_{0}^{\infty} \cdots \int_{0}^{\infty} \frac{1}{\left(u_{1}+\cdots u_{i-1}+1+u_{i+1}+\cdots+u_{n}\right)^{\lambda}} \\
& \times \prod_{j=1(j \neq i)}^{n} u_{j}^{\frac{\lambda}{r_{j}}-1} d u_{1} \cdots d u_{i-1} d u_{i+1} \cdots d u_{n} .
\end{aligned}
$$

Then by Lemma 9.15 and (9.1.19) (cf. [2], p. 341-342), we obtain (8).

The lemma is proved. 
Lemma 4 We have the following inequality:

$$
\begin{aligned}
H_{\lambda} & :=\int_{0}^{\infty} \cdots \int_{0}^{\infty} \frac{1}{\left(\sum_{i=1}^{n} x_{i}\right)^{\lambda}} \prod_{i=1}^{n} F_{i}\left(x_{i}\right) d x_{1} \cdots d x_{n} \\
& <\prod_{i=1}^{n}\left[k_{\lambda}^{(i)} \int_{0}^{\infty} x_{i}^{p_{i}\left(1-\frac{\lambda}{r_{i}}\right)-c_{\lambda}-1} F_{i}^{p_{i}}\left(x_{i}\right) d x_{i}\right]^{\frac{1}{p_{i}}} .
\end{aligned}
$$

Proof By (6) and Hölder's integral inequality (cf. [39]), we obtain

$$
\begin{aligned}
H_{\lambda}= & \int_{0}^{\infty} \cdots \int_{0}^{\infty} \frac{1}{\left(\sum_{i=1}^{n} x_{i}\right)^{\lambda}} \prod_{i=1}^{n}\left[x_{i}^{\left(\frac{\lambda}{r_{i}}-1\right)\left(1-p_{i}\right)} \prod_{j=1(j \neq i)}^{n} x_{j}^{\frac{\lambda}{r_{j}}-1}\right]^{\frac{1}{p_{i}}} F_{i}\left(x_{i}\right) d x_{1} \cdots d x_{n} \\
\leq & \prod_{i=1}^{n}\left\{\int_{0}^{\infty}\left[\int_{0}^{\infty} \cdots \int_{0}^{\infty} \frac{1}{\left(\sum_{i=1}^{n} x_{i}\right)^{\lambda}} x_{i}^{\frac{\lambda}{r_{i}}+c_{\lambda}} \prod_{j=1(j \neq i)}^{n} x_{j}^{\frac{\lambda}{r_{j}}-1} d x_{1} \cdots d x_{i-1} d x_{i+1} \cdots d x_{n}\right]\right. \\
& \left.\times x_{i}^{p_{i}\left(1-\frac{\lambda}{r_{i}}\right)-c_{\lambda}-1} F_{i}^{p_{i}}\left(x_{i}\right) d x_{i}\right\} \\
= & \prod_{i=1}^{n}\left[\int_{0}^{\frac{1}{p_{i}}} \omega_{\lambda}^{(i)}\left(x_{i}\right) x_{i}^{p_{i}\left(1-\frac{\lambda}{r_{i}}\right)-c_{\lambda}-1} F_{i}^{p_{i}}\left(x_{i}\right) d x_{i}\right]^{\frac{1}{p_{i}}} .
\end{aligned}
$$

If (11) takes the form of an equality, then there exist constants $C_{i}, C_{k}(i \neq k)$ such that they are not all zero and

$$
\begin{aligned}
C_{i} x_{i}^{\frac{\lambda}{r_{i}}+c_{\lambda}} \prod_{j=1(j \neq i)}^{n} x_{j}^{\frac{\lambda}{r_{j}}-1} x_{i}^{p_{i}\left(1-\frac{\lambda}{r_{i}}\right)-c_{\lambda}-1} F_{i}^{p_{i}}\left(x_{i}\right) \\
=C_{k} x_{k}^{\frac{\lambda}{r_{i}}+c_{\lambda}} \prod_{j=1(j \neq k)}^{n} x_{j}^{\frac{\lambda}{r_{j}}-1} x_{k}^{p_{k}\left(1-\frac{\lambda}{r_{k}}\right)-c_{\lambda}-1} F_{k}^{p_{k}}\left(x_{k}\right) \quad \text { a.e. in } \mathrm{R}_{+} .
\end{aligned}
$$

namely, $C_{i} x_{i}^{p_{i}\left(1-\frac{\lambda}{r_{i}}\right)} F_{i}^{p_{i}}\left(x_{i}\right)=C_{k} x_{k}^{p_{k}\left(1-\frac{\lambda}{r_{k}}\right)} F_{k}^{p_{k}}\left(x_{k}\right)=C$ a.e. in $\mathrm{R}_{+}$. Assuming that $C_{i} \neq 0$, we have

$$
x_{i}^{p_{i}\left(1-\frac{\lambda}{r_{i}}\right)-c_{\lambda}-1} F_{i}^{p_{i}}\left(x_{i}\right)=\frac{C}{C_{i}} x_{i}^{-c_{\lambda}-1} \quad \text { a.e. in } \mathrm{R}_{+},
$$

which contradicts the fact that $0<\int_{0}^{\infty} x_{i}^{p_{i}\left(1-\frac{\lambda}{r_{i}}\right)-c_{\lambda}-1} F_{i}^{p_{i}}\left(x_{i}\right) d x_{i}<\infty$, in view of $\int_{0}^{\infty} x_{i}^{-c_{\lambda}-1} d x_{i}=\infty$. Then by (8) and (11), we have (10).

The lemma is proved.

Remark 1 Replacing $\lambda$ (resp. $\frac{\lambda}{r_{i}}$ ) by $\lambda+n$ (resp. $\frac{\lambda}{r_{i}}+1$ ) in (10), we have

$$
\begin{aligned}
H_{\lambda+n} & =\int_{0}^{\infty} \cdots \int_{0}^{\infty} \frac{1}{\left(\sum_{i=1}^{n} x_{i}\right)^{\lambda+n}} \prod_{i=1}^{n} F_{i}\left(x_{i}\right) d x_{1} \cdots d x_{n} \\
& <\prod_{i=1}^{n}\left(\tilde{k}_{\lambda+n}^{(i)} \int_{0}^{\infty} x_{i}^{-p_{i} \frac{\lambda}{r_{i}}-c_{\lambda}-1} F_{i}^{p_{i}}\left(x_{i}\right) d x_{i}\right)^{\frac{1}{p_{i}}},
\end{aligned}
$$


where we denote

$$
\tilde{k}_{\lambda+n}^{(i)}:=\frac{\Gamma\left(\lambda\left(1-\frac{1}{r_{i}}\right)+n-1\right)}{\Gamma\left(\sum_{j=1(j \neq i)}^{n}\left(\frac{\lambda}{r_{j}}+1\right)\right)} \cdot \frac{\prod_{j=1}^{n} \Gamma\left(\frac{\lambda}{r_{j}}+1\right)}{\Gamma(\lambda+n)} \in \mathrm{R}_{+} \quad(i=1, \ldots, n) .
$$

\section{Main results and a corollary}

Theorem 1 We have the following inequality:

$$
\begin{aligned}
I: & =\int_{0}^{\infty} \cdots \int_{0}^{\infty} \frac{1}{\left(\sum_{i=1}^{n} x_{i}\right)^{\lambda}} \prod_{i=1}^{n} f_{i}\left(x_{i}\right) d x_{1} \cdots d x_{n} \\
& <\frac{\Gamma(\lambda+n)}{\Gamma(\lambda)} \prod_{i=1}^{n}\left(\tilde{k}_{\lambda+n}^{(i)} \int_{0}^{\infty} x_{i}^{-p_{i} \frac{\lambda}{r_{i}}-c_{\lambda}-1} F_{i}^{p_{i}}\left(x_{i}\right) d x_{i}\right)^{\frac{1}{p_{i}}} .
\end{aligned}
$$

In particular, for $\sum_{i=1}^{n} \frac{1}{r_{i}}=1$, we have

$$
0<\int_{0}^{\infty} x_{i}^{-p_{i} \frac{\lambda}{r_{i}}-1} F_{i}^{p_{i}}\left(x_{i}\right) d x_{i}<\infty \quad(i=1, \ldots, n)
$$

and the following inequality:

$$
\begin{aligned}
I & =\int_{0}^{\infty} \cdots \int_{0}^{\infty} \frac{1}{\left(\sum_{i=1}^{n} x_{i}\right)^{\lambda}} \prod_{i=1}^{n} f_{i}\left(x_{i}\right) d x_{1} \cdots d x_{n} \\
& <\frac{1}{\Gamma(\lambda)} \prod_{i=1}^{n} \frac{\lambda}{r_{i}} \Gamma\left(\frac{\lambda}{r_{i}}\right)\left(\int_{0}^{\infty} x_{i}^{-p_{i} \frac{\lambda}{r_{i}}-1} F_{i}^{p_{i}}\left(x_{i}\right) d x_{i}\right)^{\frac{1}{p_{i}}} .
\end{aligned}
$$

Proof By (4) and (5), we have

$$
\begin{aligned}
I & =\frac{1}{\Gamma(\lambda)} \int_{0}^{\infty} \cdots \int_{0}^{\infty} \prod_{i=1}^{n} f_{i}\left(x_{i}\right) \int_{0}^{\infty} t^{\lambda-1} e^{-t\left(x_{1}+\cdots+x_{n}\right)} d t d x_{1} \cdots d x_{n} \\
& =\frac{1}{\Gamma(\lambda)} \int_{0}^{\infty} t^{\lambda-1} \prod_{i=1}^{n} \int_{0}^{\infty} e^{-t x_{i}} f_{i}\left(x_{i}\right) d x_{i} d t \\
& =\frac{1}{\Gamma(\lambda)} \int_{0}^{\infty} t^{\lambda+n-1} \prod_{i=1}^{n} \int_{0}^{\infty} e^{-t x_{i}} F_{i}\left(x_{i}\right) d x_{i} d t \\
& =\frac{1}{\Gamma(\lambda)} \int_{0}^{\infty} \cdots \int_{0}^{\infty} \prod_{i=1}^{n} F_{i}\left(x_{i}\right) \int_{0}^{\infty} t^{\lambda+n-1} e^{-t\left(x_{1}+\cdots+x_{n}\right)} d t d x_{1} \cdots d x_{n} \\
& =\frac{\Gamma(\lambda+n)}{\Gamma(\lambda)} H_{\lambda+n} .
\end{aligned}
$$

Then by (12), we have (13).

The theorem is proved.

Theorem 2 The constant factor $\frac{1}{\Gamma(\lambda)} \prod_{i=1}^{n} \frac{\lambda}{r_{i}} \Gamma\left(\frac{\lambda}{r_{i}}\right)$ in (14) is the best possible. 
Proof For any $0<\varepsilon<\lambda \min _{1 \leq i \leq n}\left\{\frac{p_{i}}{r_{i}}\right\}$, we set

$$
\tilde{f}_{i}\left(x_{i}\right):=\left\{\begin{array}{ll}
0, & 0<x_{i} \leq 1, \\
x_{i}^{\frac{\lambda}{r_{i}}-\frac{\varepsilon}{p_{i}}-1}, & x_{i}>1,
\end{array} \quad(i=1, \ldots, n) .\right.
$$

We obtain that $\tilde{f}_{i}\left(x_{i}\right)=o\left(e^{t x_{i}}\right)\left(t>0 ; x_{i} \rightarrow \infty\right)$, and $\tilde{F}_{i}\left(x_{i}\right) \equiv 0\left(0<x_{i} \leq 1\right)$,

$$
\tilde{F}_{i}\left(x_{i}\right)=\int_{0}^{x_{i}} \tilde{f}_{i}(t) d t=\int_{1}^{x_{i}} t^{\frac{\lambda}{r_{i}}-\frac{\varepsilon}{p_{i}}-1} d t=\frac{x_{i}^{\frac{\lambda}{r_{i}}-\frac{\varepsilon}{p_{i}}}-1}{\frac{\lambda}{r_{i}}-\frac{\varepsilon}{p_{i}}}<\frac{x_{i}^{\frac{\lambda}{r_{i}}-\frac{\varepsilon}{p_{i}}}}{\frac{\lambda}{r_{i}}-\frac{\varepsilon}{p_{i}}} \quad\left(x_{i}>1 ; i=1, \ldots, n\right) .
$$

If there exists a positive constant $M\left(M \leq \frac{1}{\Gamma(\lambda)} \prod_{i=1}^{n} \frac{\lambda}{r_{i}} \Gamma\left(\frac{\lambda}{r_{i}}\right)\right)$ such that (14) is valid when replacing $\frac{1}{\Gamma(\lambda)} \prod_{i=1}^{n} \frac{\lambda}{r_{i}} \Gamma\left(\frac{\lambda}{r_{i}}\right)$ by $M$, then in particular, by substitution of $f_{i}\left(x_{i}\right)=$ $\tilde{f}_{i}\left(x_{i}\right)$ and $F_{i}\left(x_{i}\right)=\tilde{F}_{i}\left(x_{i}\right)$, we have

$$
\begin{aligned}
\tilde{I} & :=\int_{0}^{\infty} \cdots \int_{0}^{\infty} \frac{1}{\left(\sum_{i=1}^{n} x_{i}\right)^{\lambda}} \prod_{i=1}^{n} \tilde{f}_{i}\left(x_{i}\right) d x_{1} \cdots d x_{n} \\
& <M \prod_{i=1}^{n}\left(\int_{0}^{\infty} x_{i}^{-p_{i} \frac{\lambda}{r_{i}}-1} \tilde{F}_{i}^{p_{i}}\left(x_{i}\right) d x_{i}\right)^{\frac{1}{p_{i}}} \\
& =M \prod_{i=1}^{n} \frac{1}{\frac{\lambda}{r_{i}}-\frac{\varepsilon}{p_{i}}}\left(\int_{1}^{\infty} x_{i}^{-\varepsilon-1} d x_{i}\right)^{\frac{1}{p_{i}}}=\frac{M}{\varepsilon} \prod_{i=1}^{n} \frac{1}{\frac{\lambda}{r_{i}}-\frac{\varepsilon}{p_{i}}} .
\end{aligned}
$$

In view of Lemma 9.1.4 (9.1.5) in [2], we find

$$
I_{\varepsilon}:=\varepsilon \tilde{I}=\varepsilon \int_{1}^{\infty} \cdots \int_{1}^{\infty} \frac{1}{\left(\sum_{i=1}^{n} x_{i}\right)^{\lambda}} \prod_{i=1}^{n} x_{i}^{\frac{\lambda}{r_{i}}-\frac{\varepsilon}{p_{i}}-1} d x_{1} \cdots d x_{n}=k_{\lambda}+o(1) \quad\left(\varepsilon \rightarrow 0^{+}\right) .
$$

Hence, we have

$$
\frac{1}{\Gamma(\lambda)} \prod_{i=1}^{n} \Gamma\left(\frac{\lambda}{r_{i}}\right)+o(1)=k_{\lambda}+o(1)=\varepsilon \tilde{I}<M \prod_{i=1}^{n} \frac{1}{\frac{\lambda}{r_{i}}-\frac{\varepsilon}{p_{i}}} .
$$

For $\varepsilon \rightarrow 0^{+}$, we find

$$
\frac{1}{\Gamma(\lambda)} \prod_{i=1}^{n} \frac{\lambda}{r_{i}} \Gamma\left(\frac{\lambda}{r_{i}}\right) \leq M
$$

which yields that the constant factor $M=\frac{1}{\Gamma(\lambda)} \prod_{i=1}^{n} \frac{\lambda}{r_{i}} \Gamma\left(\frac{\lambda}{r_{i}}\right)$ in (14) is the best possible.

The theorem is proved.

Setting $x=\frac{1}{x_{1}}, f(x)=x^{\lambda-2} f_{1}\left(\frac{1}{x}\right)$ in $I$ of $(14)$, we have

$$
I=\int_{0}^{\infty} \cdots \int_{0}^{\infty} \frac{f(x)}{\left(1+\sum_{i=2}^{n} x x_{i}\right)^{\lambda}} \prod_{i=2}^{n} f_{i}\left(x_{i}\right) d x d x_{2} \cdots d x_{n} .
$$


For $f_{1}(t)=t^{\lambda-2} f\left(\frac{1}{t}\right)$, we find

$$
F_{1}\left(x_{1}\right)=\int_{0}^{x_{1}} f_{1}(t) d t=\int_{0}^{x_{1}} t^{\lambda-2} f\left(\frac{1}{t}\right) d t
$$

Then, replacing back $x($ resp. $f(x))$ by $x_{1}\left(\right.$ resp. $\left.f_{1}\left(x_{1}\right)\right)$, we have

Corollary 1 If $\tilde{F}_{1}\left(x_{1}\right)=\int_{0}^{x_{1}} t^{\lambda-2} f_{1}\left(\frac{1}{t}\right) d t$,

$$
\tilde{F}_{i}\left(x_{i}\right):=\int_{0}^{x_{i}} f_{i}(t) d t \quad(i=2, \ldots, n)
$$

then we have the following inequality with the nonhomogeneous kernel:

$$
\begin{gathered}
\int_{0}^{\infty} \cdots \int_{0}^{\infty} \frac{1}{\left(1+\sum_{i=2}^{n} x_{1} x_{i}\right)^{\lambda}} \prod_{i=1}^{n} f_{i}\left(x_{i}\right) d x_{1} \cdots d x_{n} \\
<\frac{1}{\Gamma(\lambda)} \prod_{i=1}^{n} \frac{\lambda}{r_{i}} \Gamma\left(\frac{\lambda}{r_{i}}\right)\left(\int_{0}^{\infty} x_{i}^{-p_{i} \frac{\lambda}{r_{i}}-1} \tilde{F}_{i}^{p_{i}}\left(x_{i}\right) d x_{i}\right)^{\frac{1}{p_{i}}},
\end{gathered}
$$

where the constant factor $\frac{1}{\Gamma(\lambda)} \prod_{i=1}^{n} \frac{\lambda}{r_{i}} \Gamma\left(\frac{\lambda}{r_{i}}\right)$ in (15) is the best possible.

Remark 2 (i) For $n=2$, (14) reduces to (cf. [40])

$$
\begin{aligned}
\int_{0}^{\infty} & \int_{0}^{\infty} \frac{f_{1}\left(x_{1}\right) f_{2}\left(x_{2}\right)}{\left(x_{1}+x_{2}\right)^{\lambda}} d x_{1} d x_{2} \\
& <\frac{\lambda^{2}}{r_{1} r_{2}} B\left(\frac{\lambda}{r_{1}}, \frac{\lambda}{r_{2}}\right)\left(\int_{0}^{\infty} x_{1}{ }^{-p_{1} \frac{\lambda}{r_{1}}-1} F_{1}^{p_{1}}\left(x_{1}\right) d x_{1}\right)^{\frac{1}{p_{1}}}\left(\int_{0}^{\infty} x_{2}{ }^{-p_{2} \frac{\lambda}{r_{2}}-1} F_{2}^{p_{2}}\left(x_{2}\right) d x_{2}\right)^{\frac{1}{p_{2}}},
\end{aligned}
$$

and (15) reduces to the following new inequality:

$$
\begin{aligned}
\int_{0}^{\infty} & \int_{0}^{\infty} \frac{f_{1}\left(x_{1}\right) f_{2}\left(x_{2}\right)}{\left(1+x_{1} x_{2}\right)^{\lambda}} d x_{1} d x_{2} \\
& <\frac{\lambda^{2}}{r_{1} r_{2}} B\left(\frac{\lambda}{r_{1}}, \frac{\lambda}{r_{2}}\right)\left(\int_{0}^{\infty} x_{1}^{-p_{1} \frac{\lambda}{r_{1}}-1} \tilde{F}_{1}^{p_{1}}\left(x_{1}\right) d x_{1}\right)^{\frac{1}{p_{1}}}\left(\int_{0}^{\infty} x_{2}^{-p_{2} \frac{\lambda}{r_{2}}-1} \tilde{F}_{2}^{p_{2}}\left(x_{2}\right) d x_{2}\right)^{\frac{1}{p_{2}}} .
\end{aligned}
$$

(ii) For $r_{i}=p_{i}(i=1, \ldots, n),(14)$ reduces to

$$
\begin{aligned}
& \int_{0}^{\infty} \cdots \int_{0}^{\infty} \frac{1}{\left(\sum_{i=1}^{n} x_{i}\right)^{\lambda}} \prod_{i=1}^{n} f_{i}\left(x_{i}\right) d x_{1} \cdots d x_{n} \\
& <\frac{1}{\Gamma(\lambda)} \prod_{i=1}^{n} \frac{\lambda}{p_{i}} \Gamma\left(\frac{\lambda}{p_{i}}\right)\left(\int_{0}^{\infty} x_{i}^{-\lambda-1} F_{i}^{p_{i}}\left(x_{i}\right) d x_{i}\right)^{\frac{1}{p_{i}}},
\end{aligned}
$$

and (15) reduces to

$$
\int_{0}^{\infty} \cdots \int_{0}^{\infty} \frac{1}{\left(1+\sum_{i=2}^{n} x_{1} x_{i}\right)^{\lambda}} \prod_{i=1}^{n} f_{i}\left(x_{i}\right) d x_{1} \cdots d x_{n}
$$




$$
<\frac{1}{\Gamma(\lambda)} \prod_{i=1}^{n} \frac{\lambda}{p_{i}} \Gamma\left(\frac{\lambda}{p_{i}}\right)\left(\int_{0}^{\infty} x_{i}^{-\lambda-1} \tilde{F}_{i}^{p_{i}}\left(x_{i}\right) d x_{i}\right)^{\frac{1}{p_{i}}} .
$$

The constant factors in the above inequalities are the best possible.

\section{Conclusions}

In this paper, following the idea of [21], by the use of the weight functions, the way of introducing parameters and the technique of real analysis, a new multiple Hilbert-type integral inequality with the kernel $\frac{1}{\left(x_{1}+\cdots+x_{n}\right)^{\lambda}}(\lambda>0)$ involving the upper limit functions is given in Theorem 1 . In a condition, the best possible constant factor related to the gamma function and a few parameters is proved in Theorem 2. A corollary about the case of nonhomogeneous kernel and some particular inequalities are obtained in Corollary 1 and Remark 2 . The lemmas and theorems provide an extensive account of this type of inequalities.

Acknowledgements

The authors thank the referee for his useful propose to reform the paper.

Funding

This work is supported by the National Natural Science Foundation (No. 61772140), and Characteristic innovation project of Guangdong Provincial Colleges and universities in 2020 (No. 2020KTSCX088). We are grateful for their help.

\section{Availability of data and materials}

The data used to support the findings of this study are included within the article.

\section{Competing interests}

The authors declare that they have no competing interests.

\section{Authors' contributions}

BY carried out the mathematical studies, participated in the sequence alignment and drafted the manuscript. JZ participated in the design of the study and performed the numerical analysis. All authors read and approved the final manuscript.

\section{Publisher's Note}

Springer Nature remains neutral with regard to jurisdictional claims in published maps and institutional affiliations.

Received: 2 October 2020 Accepted: 8 January 2021 Published online: 19 January 2021

\section{References}

1. Hardy, G.H., Littlewood, J.E., Polya, G.: Inequalities. Cambridge University Press, Cambridge (1934)

2. Yang, B.C.: The Norm of Operator and Hilbert-Type Inequalities. Science Press, Beijing (2009)

3. Yang, B.C.: Hilbert-Type Integral Inequalities. Bentham Science, The United Arab Emirates (2009)

4. Yang, B.C.: On the norm of an integral operator and applications. J. Math. Anal. Appl. 321, 182-192 (2006)

5. Xu, J.S.: Hardy-Hilbert's inequalities with two parameters. Adv. Math. 36(2), 63-76 (2007)

6. Yang, B.C.: On the norm of a Hilbert's type linear operator and applications. J. Math. Anal. Appl. 325, $529-541$ (2007)

7. Xie, Z.T., Zeng, Z., Sun, Y.F.: A new Hilbert-type inequality with the homogeneous kernel of degree -2. Adv. Appl. Math. Sci. 12(7), 391-401 (2013)

8. Zhen, Z., Raja Rama Gandhi, K., Xie, Z.T.: A new Hilbert-type inequality with the homogeneous kernel of degree -2 and with the integral. Bull. Math. Sci. Appl. 3(1), 11-20 (2014)

9. Xin, D.M.: A Hilbert-type integral inequality with the homogeneous kernel of zero degree. Math. Theory Appl. 30(2), 70-74 (2010)

10. Azar, L.E.: The connection between Hilbert and Hardy inequalities. J. Inequal. Appl. 2013, 452 (2013)

11. Batbold, T., Sawano, Y.: Sharp bounds for $m$-linear Hilbert-type operators on the weighted Morrey spaces. Math. Inequal. Appl. 20, 263-283 (2017)

12. Adiyasuren, V., Batbold, T., Krnic, M.: Multiple Hilbert-type inequalities involving some differential operators. Banach J. Math. Anal. 10, 320-337 (2016)

13. Adiyasuren, V., Batbold, T., Krnić, M.: Hilbert-type inequalities involving differential operators, the best constants and applications. Math. Inequal. Appl. 18, 111-124 (2015)

14. Rassias, M., Yang, B.C.: On half-discrete Hilbert's inequality. Appl. Math. Comput. 220, 75-93 (2013)

15. Yang, B.C., Krnic, M.: A half-discrete Hilbert-type inequality with a general homogeneous kernel of degree 0 . J. Math. Inequal. 6(3), 401-417 (2012)

16. Rassias, M., Yang, B.C.: A multidimensional half-discrete Hilbert-type inequality and the Riemann zeta function. Appl. Math. Comput. 225, 263-277 (2013) 
17. Rassias, M., Yang, B.C.: On a multidimensional half-discrete Hilbert-type inequality related to the hyperbolic cotangent function. Appl. Math. Comput. 242, 800-813 (2013)

18. Huang, Z.X., Yang, B.C.: On a half-discrete Hilbert-type inequality similar to Mulholland's inequality. J. Inequal. Appl. 2013, $290(2013)$

19. Yang, B.C., Lebnath, L.: Half-Discrete Hilbert-Type Inequalities. World Scientific, Singapore (2014)

20. Krnic, M., Pecaric, J.: Extension of Hilbert's inequality. J. Math. Anal. Appl. 324(1), 150-160 (2006)

21. Adiyasuren, V., Batbold, T., Azar, L.E.: A new discrete Hilbert-type inequality involving partial sums. J. Inequal. Appl. 2019, $127(2019)$

22. Hong, Y., Wen, Y.: A necessary and sufficient condition of that Hilbert type series inequality with homogeneous kernel has the best constant factor. Ann. Math. 37A(3), 329-336 (2016)

23. Hong, Y:: On the structure character of Hilbert's type integral inequality with homogeneous kernel and applications. J. Jilin Univ. Sci. Ed. 55(2), 189-194 (2017)

24. Hong, Y., Huang, Q.L., Yang, B.C., Liao, J.L.: The necessary and sufficient conditions for the existence of a kind of Hilbert-type multiple integral inequality with the non-homogeneous kernel and its applications. J. Inequal. Appl. 2017, $316(2017)$

25. Xin, D.M., Yang, B.C., Wang, A.Z.: Equivalent property of a Hilbert-type integral inequality related to the beta function in the whole plane. J. Funct. Spaces 2018, Article ID 2691816 (2018)

26. Hong, Y., He, B., Yang, B.C.: Necessary and sufficient conditions for the validity of Hilbert type integral inequalities with a class of quasi-homogeneous kernels and its application in operator theory. J. Math. Inequal. 12(3), 777-788 (2018)

27. Rassias, M., Yang, B.C.: On an equivalent property of a reverse Hilbert-type integral inequality related to the extended Hurwitz-zeta function. J. Math. Inequal. 13(2), 315-334 (2019)

28. Rassias, M., Yang, B.C.: A reverse Mulholland-type inequality in the whole plane with multi-parameters. Appl. Anal. Discrete Math. 13, 290-308 (2019)

29. Luo, R.C., Yang, B.C.: Parameterized discrete Hilbert-type inequalities with intermediate variables. J. Inequal. Appl. 2019, $142(2019)$

30. He, L.P., Liu, H.Y., Yang, B.C.: Parametric Mulholland-type inequalities. J. Appl. Anal. Comput. 9(5), 1973-1986 (2019)

31. Yang, B.C., Wu, S.H., Wang, A.Z.: On a reverse half-discrete Hardy-Hilbert's inequality with parameters. Mathematics 7 , 1054 (2019)

32. Yang, B.C., Wu, S.H., Chen, Q.: On an extended Hardy-Littlewood-Polya's inequality. AIMS Math. 5(2), 1550-1561 (2020)

33. Yang, B.C., Huang, M.F., Zhong, Y.R.: Equivalent statements of a more accurate extended Mulholland's inequality with a best possible constant factor. Math. Inequal. Appl. 23(1), 231-244 (2020)

34. Yang, B.C., Wu, S.H., Wang, A.Z: A new Hilbert-type inequality with positive homogeneous kernel and its equivalent form. Symmetric 12, 342 (2020). https://doi.org/10.3390/sym12030342

35. Liao, J.Q., Hong, Y., Yang, B.C.: Equivalent conditions of a Hilbert-type multiple integral inequality holding. J. Funct. Spaces 2020, Article ID 3050952 (2020)

36. Yang, B.C., Wu, S.H., Chen, Q.: A new extension of Hardy-Hilbert's inequality containing kernel of double power functions. Mathematics 8, 339 (2020). https://doi.org/10.3390/math8060894

37. Rassias, M., Yang, B.C., Raigorodskii, A.: On the reverse Hardy-type integral inequalities in the whole plane with the extended Riemann Zeta function. J. Math. Inequal. 14(2), 525-546 (2020)

38. Yang, B.C., Zhong, Y.R.: On a reverse Hardy-Littlewood-Polya's inequality. J. Appl. Anal. Comput. 10(5), 2220-2232 (2020)

39. Kuang, J.C.: Applied Inequalities. Shangdong Science and Technology Press, Jinan (2004)

40. Mo, H.M., Yang, B.C.: On a new Hilbert-type integral inequality involving the upper limit functions. J. Inequal. Appl. $2020,5(2020)$

\section{Submit your manuscript to a SpringerOpen ${ }^{0}$ journal and benefit from:}

- Convenient online submission

- Rigorous peer review

Open access: articles freely available online

- High visibility within the field

- Retaining the copyright to your article

Submit your next manuscript at $\gg$ springeropen.com 